\title{
Fast and accurate normalization of vectors and quaternions
}

\author{
Walter F. Mascarenhas \\ Instituto de Matemática e Estatística, Universidade de São Paulo, Brazil
}

\begin{abstract}
We present fast and accurate ways to normalize two and three dimensional vectors and quaternions and compute their length. Our approach is an adaptation of ideas used in the linear algebra library LAPACK, and we believe that the computational geometry and computer aided design communities are not aware of the possibility of speeding up these fundamental operations in the robust way proposed here.
\end{abstract}

Keywords: vectors, quaternions, normalization, efficiency, accuracy

\section{Introduction}

Obtaining the length of vectors and normalizing them are basic operations implemented by most computational geometry and computer aided design software libraries. In this case, for $n=2$ or 3 , given a vector $\mathbf{x} \in \mathbb{R}^{n} \backslash\{0\}$ we compute

$$
\|\mathbf{x}\|:=\sqrt{x_{1}^{2}+\cdots+x_{n}^{2}} \quad \text { and } \quad \overline{\mathbf{x}}:=\left(\frac{x_{1}}{r}, \ldots, \frac{x_{n}}{r}\right),
$$

so that $\overline{\mathbf{x}}$ has length one and can be used in geometric primitives, like the computation of the distance of a point to a line or a plane. In the ObjectARX ${ }^{\circledR}$ library [7] for example, vectors have a normalize method and most libraries for computational geometry and CAD have similar functions.

Sums of squares are also required for dealing with quaternions, which are used to represent rotations in three dimensions. In fact, when we represent rotations by quaternions we either assume that they are normalized or perform a division by the square of their norm. Therefore, when dealing with a quaternion $\mathbf{q}:=\left(x_{1}, x_{2}, x_{3}, x_{4}\right)$

$$
x_{1}^{2}+x_{2}^{2}+x_{3}^{2}+x_{4}^{2} \quad \text { or } \quad r(\mathbf{q}):=\|\mathbf{q}\|:=\sqrt{x_{1}^{2}+x_{2}^{2}+x_{3}^{2}+x_{4}^{2}},
$$

and even when we only apply rotations with normalized quaternions we must evaluate the expressions in Equation (2) in the preliminary step in which we compute them.

Email address: walter.mascarenhas@gmail.com (Walter F. Mascarenhas)

${ }^{1}$ Depto. de Computa cão, IME USP, Cidade Universitária, Rua do Matão 1010, São Paulo SP, Brazil. CEP 05508-090, Tel.: +55-11-3091 5411, Fax: +55-11-30916134 
This note presents efficient and accurate algorithms to evaluate the expressions in Equations (1)-(2). In theory, evaluating these expressions is easy, we need only to pay attention to $\mathbf{x}=0$. The robust implementation of these expressions in finite precision arithmetic is also well known among experts in computational geometry. For example, we wrote to a respected scholar in this field and received a precise answer, explaining that actually normalization of vectors is a bit tricky, because when $x_{1}$ is too small the computed value $\mathrm{fl}\left(x_{1}^{2}\right)$ may be zero even when $x_{1}$ is different from zero, and similarly $\mathrm{fl}\left(x_{1}^{2}\right)$ may overflow when $x_{1}$ is large. The authors of professional software libraries are also well aware of these problems. The documentation of the ObjectARX ${ }^{\circledR}$ library for instance states clearly that the normalization may fail when the vector $\mathbf{x}$ is too small.

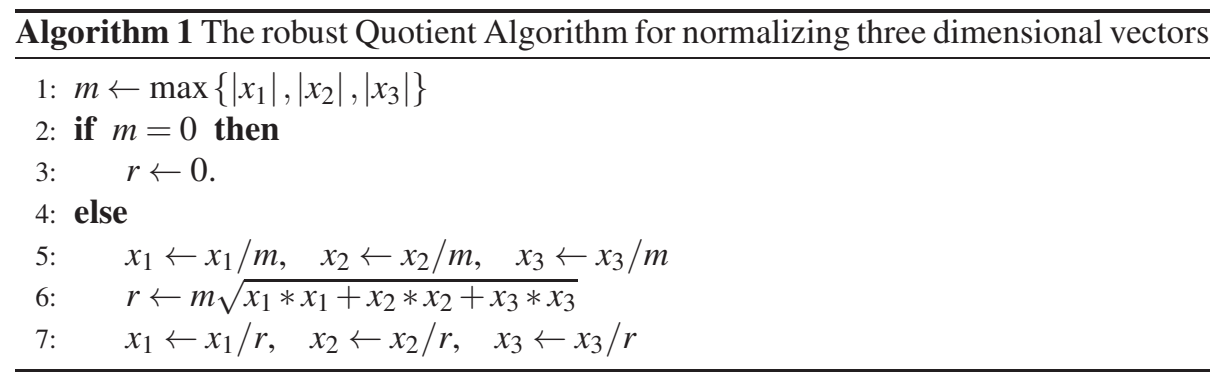

The scholar mentioned that Algorithm 1 above would handle overflow, and we could also use Algorithm 2 below. These algorithms use divisions, and we call them "quotient algorithms." When the input is finite, they only fail due to overflow, in situations in which their input warrants this result. Moreover, Algorithm 2 returns NaNs (not a numbers) when the input contains NaNs.

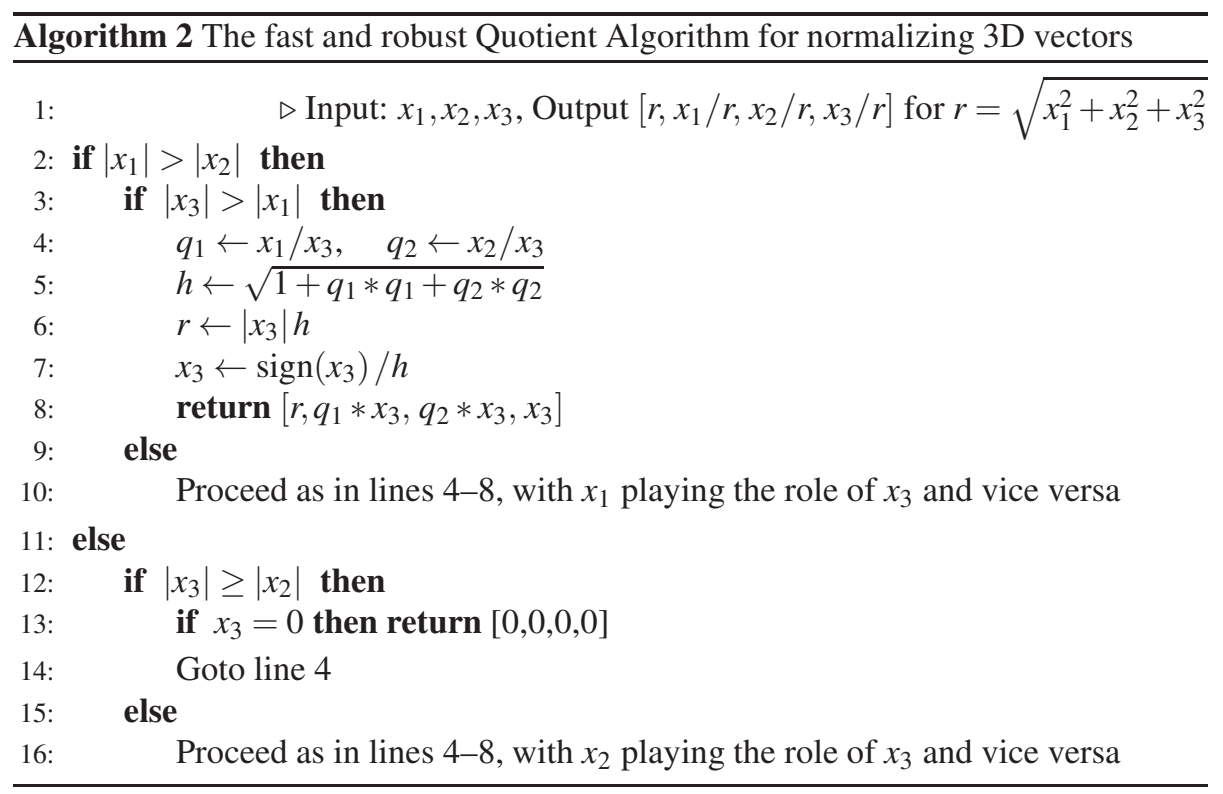


We have been using Algorithm 2 for many years, but found recently that by adapting the method proposed by Bindel, Demmel, Kahan and Marques in [2] to compute plane rotations we obtain a better way to evaluate the expressions in Equations (1)-(2). Their method uses scaling instead of divisions and leads to the "scaling algorithms" presented in this article. The superiority of scaling algorithms to quotient algorithms is illustrated by Table A.3 in Appendix A. The degree by which a scaling algorithm is superior to the corresponding quotient algorithm in the entries of Table A.3 varies, but cases in which the scaling algorithm is twice as fast as the quotient algorithm are not uncommon. Moreover, there is no instance in which the scaling algorithm is slower, and we prove here that the rounding errors incurred by scaling algorithms are small.

One may ask whether it wouldn't be simpler to code Equations (1)-(2) in the obvious way. This question is relevant, specially when one knows that underflow and overflow will not happen. Table A.4 in Appendix A may help one to decide whether the robustness brought by scaling is worth its cost. This table compares the algorithms described here with the naive algorithm. On the one hand, it shows that scaling can be expensive. For instance, we frequently normalize quaternions which already have a norm close to one, and the last two rows of Table A.4 show that scaling is not worth in this case. On the other hand, Table A.4 also shows that, depending on the compiler and the precision, there are cases in which the overhead due to scaling is minimal.

In the rest of this note we present scaling algorithms to normalize vectors and quaternions and compute their length, and describe their numerical properties. Section 3 describes scaling algorithms for normalizing vectors and computing their norm, and presents formal results about the numerical properties of these algorithms. Section 4 does the same for quaternions. Appendix A contains the experimental results and in Appendix B we prove that our estimates for the rounding errors are correct.

\section{Accurate and efficient scaling}

Quotient and scaling algorithms have the same motivation: to avoid underflow and overflow in the evaluation of

$$
x_{1}^{2}+x_{2}^{2}, \quad x_{1}^{2}+x_{2}^{2}+x_{3}^{2} \quad \text { and } \quad x_{1}^{2}+x_{2}^{2}+x_{3}^{2}+x_{4}^{2} .
$$

They both evaluate $m:=\max \left|x_{k}\right|$, but use $m$ differently. Quotient algorithms divide $\mathbf{x}$ by $m$, and are inefficient because divisions are much more expensive than sums and multiplications. Scaling algorithms perform the minimal work required to avoid underflow and overflow in Equation (3). They use floating point numbers $\tau_{\min }$ and $\tau_{\max }$ such that if $\tau_{\min } \leq m \leq \tau_{\max }$ then the sums of squares in (3) can be computed with a small relative error. For instance, the results about dot products in [6] show that, in a binary IEEE machine, with the parameters in Table 1 below, if

$$
\tau_{\min }:=2^{\left\lceil\left(\log _{2}\left(\alpha / u^{2}\right)\right) / 2\right\rceil} \quad \text { and } \quad \tau_{\max }:=2^{\left\lfloor\left(\log _{2}(\omega)-3\right) / 2\right\rfloor}
$$

and $\tau_{\min } \leq m \leq \tau_{\max }$ then we have $|\mathrm{fl}(\|\mathbf{x}\|)-\|\mathbf{x}\|| \leq 2.5 u\|\mathbf{x}\|$. This means that the computed value of $\|\mathbf{x}\|$, which we denote by $\mathrm{fl}(\|\mathbf{x}\|)$, has a small relative error, because in single precision $u \approx 6.0 \times 10^{-8}$ and in double precision $u \approx 1.1 \times 10^{-16}$. 
Table 1: The parameters describing a floating point arithmetic

\begin{tabular}{c|c}
\hline Parameter & Meaning \\
\hline$u$ & unit roundoff \\
$\alpha$ & the smallest positive floating point number \\
$v$ & the smallest positive normal floating point number \\
$\omega$ & the largest finite floating point number
\end{tabular}

Table 2: The parameters for the binary IEEE arithmetics

\begin{tabular}{c|cccc|cccc} 
& \multicolumn{4}{|c}{ Algorithms } & \multicolumn{5}{c}{ Hardware } \\
\hline Precision & $\tau_{\min }$ & $\sigma_{\min }$ & $\tau_{\max }$ & $\sigma_{\max }$ & $u$ & $\alpha$ & $v$ & $\omega$ \\
\hline Single & $2^{-49}$ & $2^{100}$ & $2^{62}$ & $2^{-66}$ & $\approx 10^{-7}$ & $\approx 10^{-45}$ & $\approx 10^{-38}$ & $\approx 10^{38}$ \\
Double & $2^{-482}$ & $2^{592}$ & $2^{510}$ & $2^{-514}$ & $\approx 10^{-16}$ & $\approx 10^{-324}$ & $\approx 10^{-308}$ & $\approx 10^{308}$
\end{tabular}

The key idea is to scale $\mathbf{x}$ so that $m$ falls in the favorable range $\left[\tau_{\min }, \tau_{\max }\right]$. To avoid rounding errors, we scale by multiplying by a power of the base of the floating arithmetic, as when we use the parameters in Table 2. These parameters satisfy Equations (5)-(10) and this ensures that the algorithms for normalizing vectors and quaternions in the next sections are accurate.

$$
\begin{array}{r}
\mathrm{fl}\left(v^{2}\right)=0, \quad \begin{array}{r}
u^{2} \geq 16 \alpha, \quad u \leq 10^{-6}, \\
\sigma_{\min } \text { and } \sigma_{\max } \text { are powers of } 2,
\end{array} \\
u^{2} \tau_{\min }^{2} \geq \alpha \quad \text { and } \quad 8 \tau_{\max }^{2} \leq \omega, \\
\omega \tau_{\min } \geq 1 \text { and } 3 v \tau_{\max } \leq 1, \\
\text { if } x \in\left(0, \tau_{\min }\right] \text { is a floating point number then } \sigma_{\min } x \in\left[\tau_{\min }, \tau_{\max }\right], \\
\text { if } x \in\left[\tau_{\max }, \omega\right] \text { then } \sigma_{\max } x \in\left[\tau_{\min }, \tau_{\max }\right] .
\end{array}
$$

Algorithms 3 and 4 below scale vectors, so that their largest entry falls in the interval $\left[\tau_{\min }, \tau_{\max }\right]$. These algorithms return the inverse of the scaling factor, so that the scaling can be undone afterwards. Finally, they handle the case in which $\mathbf{x}=0$ properly and propagate NaNs, in the sense that $x_{k}$ is NaN for some $k$ if and only if some of the output $x_{k}$ is $\mathrm{NaN}$. 

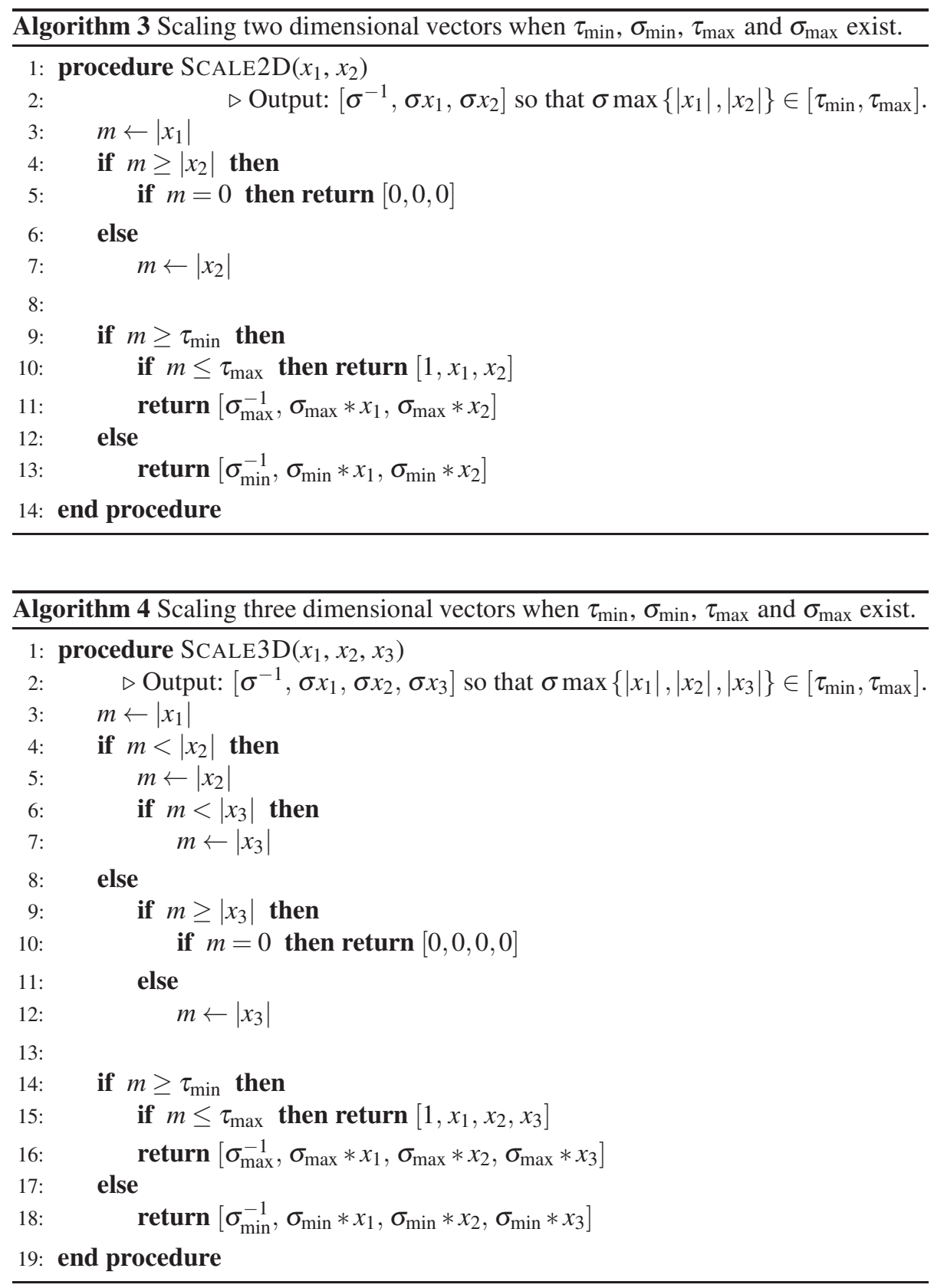

\section{Normalizing vectors}

Here we use algorithms Scale2D and Scale3D to compute the length of two and three dimensional vectors and normalize them accurately and efficiently. This com- 
putation is performed by Algorithms 5 and 6 in the end of this section. Before the algorithms we present Lemma 1, which describes their numerical properties. In summary, Lemma 1 shows that, in an IEEE machine, the scaling algorithms to normalize vectors incur in errors which are only a small multiple of the unit roundoff, both in the computed length of the vector and its direction.

Lemma 1 must be adapted for arithmetics which do not follow the IEEE standard for floating point arithmetic [4]. For instance, modern processors allow for faster modes of execution called DAZ and FTZ, which are incompatible with this standard. When algorithms are executed in DAZ mode subnormal numbers are set to zero on input to functions, and the input $\mathbf{x}$ in Lemma 1 must be considered to be what is left after subnormals are set to zero, but after this correction Lemma 1 does apply. In FTZ mode subnormal numbers are set to zero on output and the bounds on the angle in Lemma 1 would increase a bit, but would still be of order $u$. The bound (11) would apply, but in bound (12) we would need to replace $\alpha / 2$ by $v$.

Lemma 1. For $n=2$ or 3 , suppose that Equations (5)-(10) are satisfied and we execute Algorithms 5 and 6 with input $\mathbf{x} \in \mathbb{R}^{n}$ and obtain $\hat{r} \in \mathbb{R}$ and $\hat{\mathbf{x}} \in \mathbb{R}^{n}$. Let us define $r:=\|\mathbf{x}\|$ and $\phi \in[-\pi, \pi]$ as the angle between $\mathbf{x}$ and $\hat{\mathbf{x}}$. If $\mathbf{x} \neq 0$ is finite then $\hat{\mathbf{x}}$ is also finite, $\hat{r} \neq 0$,

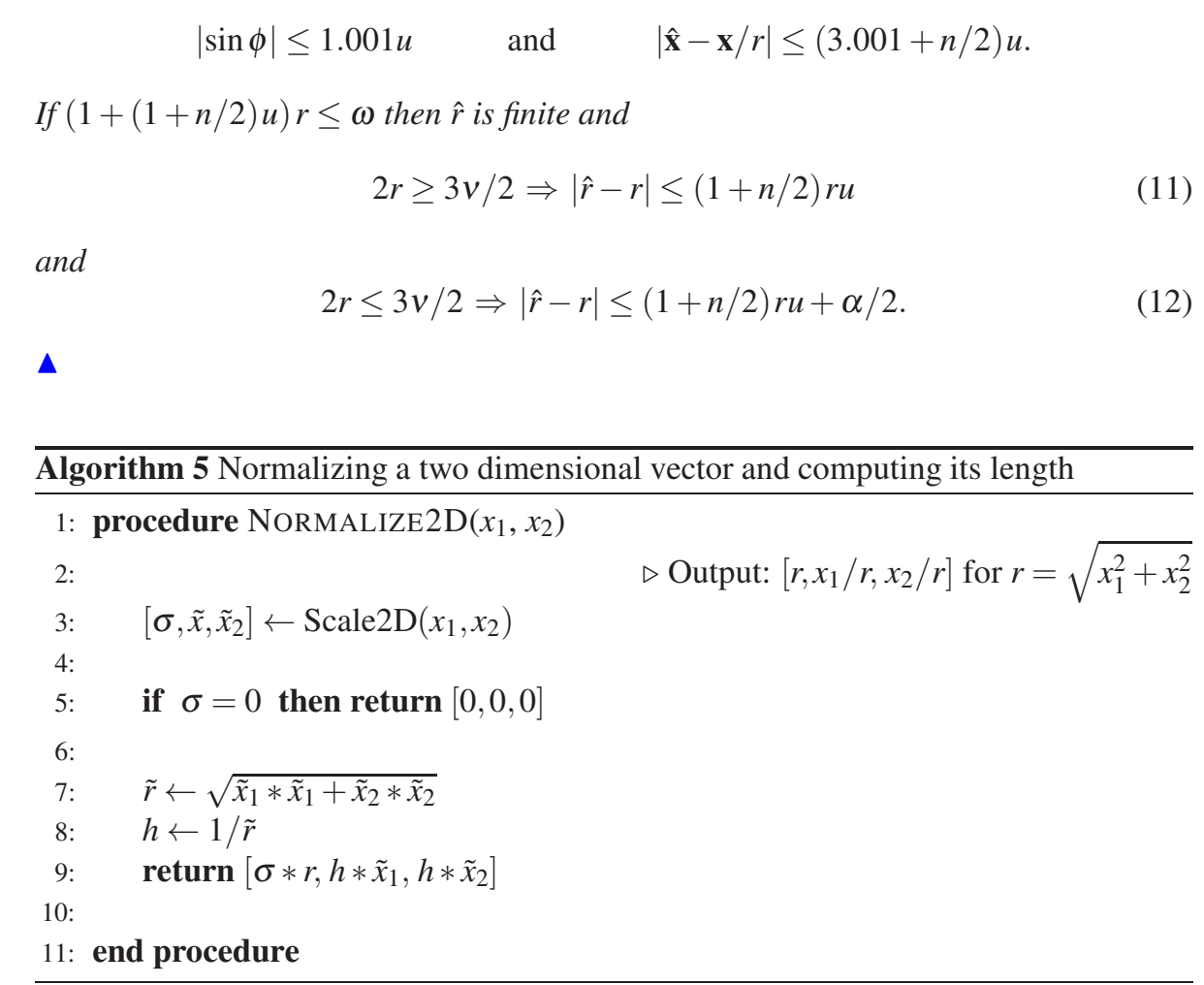




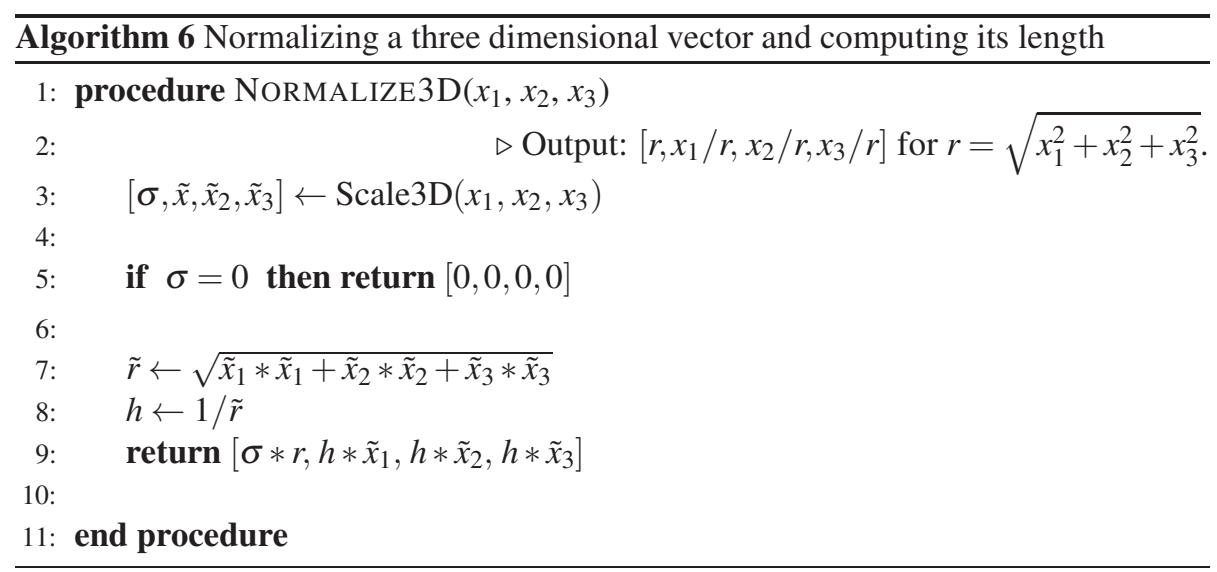

\section{Quaternions}

In this section we present an scaling algorithm to normalize quaternions. We are only concerned with the numerical aspects of computing with quaternions, and recommend Altmann's book [1] for a broader discussion about them. When the quaternion $\mathbf{q}=\left(q_{1}, q_{2}, q_{3}, q_{4}\right)$ is different from zero it is related to the rotation matrix

$$
\mathbf{R}(\mathbf{q}):=\frac{1}{\|\mathbf{q}\|^{2}}\left(\begin{array}{ccc}
q_{1}^{2}+q_{4}^{2}-q_{2}^{2}-q_{3}^{2} & 2\left(q_{1} q_{2}-q_{3} q_{4}\right) & 2\left(q_{1} q_{3}+q_{2} q_{4}\right) \\
2\left(q_{1} q_{2}+q_{3} q_{4}\right) & q_{2}^{2}+q_{4}^{2}-q_{1}^{2}-q_{3}^{2} & 2\left(q_{2} q_{3}-q_{1} q_{4}\right) \\
2\left(q_{1} q_{3}-q_{2} q_{4}\right) & 2\left(q_{2} q_{3}+q_{1} q_{4}\right) & q_{3}^{2}+q_{4}^{2}-q_{1}^{2}-q_{2}^{2}
\end{array}\right)
$$

and when $\|\mathbf{q}\|=1$ this expression simplifies to

$$
\mathbf{R}(\mathbf{q})=\left(\begin{array}{ccc}
1-2\left(q_{2}^{2}+2 q_{3}^{2}\right) & 2\left(q_{1} q_{2}-q_{3} q_{4}\right) & 2\left(q_{1} q_{3}+q_{2} q_{4}\right) \\
2\left(q_{1} q_{2}+q_{3} q_{4}\right) & 1-2\left(q_{1}^{2}+q_{3}^{2}\right) & 2\left(q_{2} q_{3}-q_{1} q_{4}\right) \\
2\left(q_{1} q_{3}-q_{2} q_{4}\right) & 2\left(q_{2} q_{3}+q_{1} q_{4}\right) & 1-2\left(q_{1}^{2}+2 q_{2}^{2}\right)
\end{array}\right) .
$$

In the end of this section we present the scaling Algorithm 7, which normalizes the quaternion $\mathbf{q}=\left(x_{1}, x_{2}, x_{3}, x_{4}\right)$ so that we can use the simplified expression for the rotation $\mathbf{R}$ above. The numerical properties of this algorithm are summarized by the following lemma, which shows that the returned quaternion is normalized up to the machine precision and the entries of the resulting matrix are also accurate, provided, of course, that $\mathbf{q}$ is finite and non zero.

Lemma 2. Suppose that Equations (5)-(10) are satisfied and we execute Algorithm 7 with input $\mathbf{q}$ and obtain $\hat{r}$ and $\hat{\mathbf{q}}$. If $\mathbf{q} \neq 0$ is finite then $\hat{\mathbf{q}}$ is also finite, $\hat{r} \neq 0$ and $\|\hat{\mathbf{q}}-\overline{\mathbf{q}}\| \leq 5.001 u$, for $\overline{\mathbf{q}}:=\mathbf{q} / r$ and $r:=\|\mathbf{q}\|$. If $(1+3 u) r \leq \omega$ then $\hat{r}$ is finite and

$$
2 r \geq 3 v / 2 \Rightarrow|\hat{r}-r| \leq 3 r u
$$

and

$$
2 r \leq 3 v / 2 \Rightarrow|\hat{r}-r| \leq 3 r u+\alpha / 2 .
$$

Moreover, for $1 \leq i, j \leq 4$,

$$
\left|\hat{q}_{i} \hat{q}_{j}-\bar{q}_{i} \bar{q}_{j}\right| \leq\left(1.001+8.001 \bar{q}_{i} \bar{q}_{j}\right) u .
$$




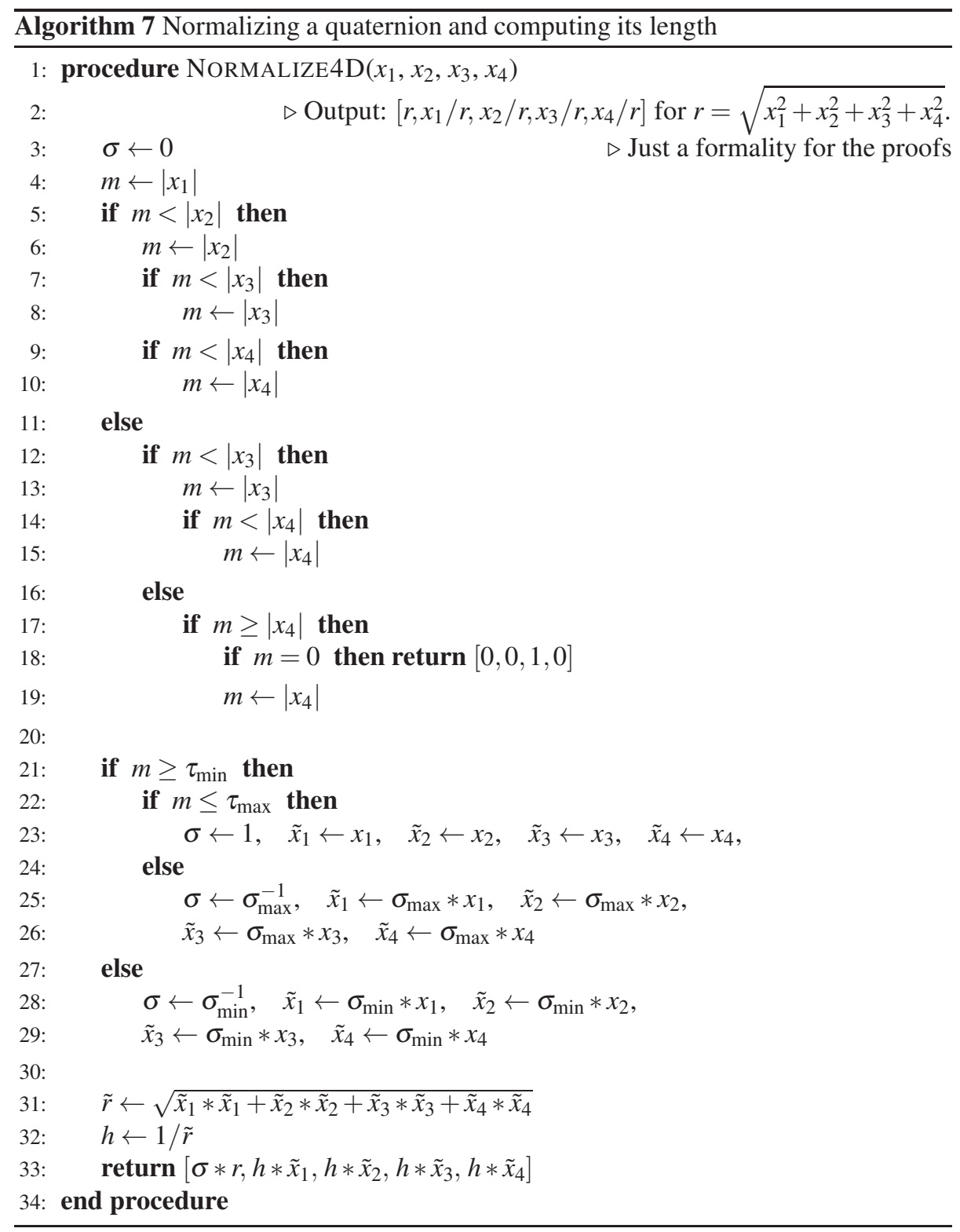


Table A.3: $\frac{\text { Time taken by a quotient algorithm }}{\text { Time taken by the corresponding scaling algorithm }}$

\begin{tabular}{cc|ccc} 
& & \multicolumn{3}{|c}{ Compiler/Operating system } \\
& GCC & Intel C++ & Visual Studio \\
Task & Precision & Linux & Linux & Windows \\
\hline Normalizing & single & $1.69 \pm 0.07$ & $1.72 \pm 0.03$ & $2.08 \pm 0.12$ \\
a 2D vector & double & $2.46 \pm 0.05$ & $1.92 \pm 0.03$ & $2.56 \pm 0.11$ \\
\hline Normalizing & single & $1.70 \pm 0.04$ & $1.95 \pm 0.03$ & $2.31 \pm 0.16$ \\
a 3D vector & double & $2.53 \pm 0.02$ & $2.59 \pm 0.08$ & $2.99 \pm 0.19$ \\
\hline Normalizing & single & $1.77 \pm 0.07$ & $1.83 \pm 0.02$ & $1.96 \pm 0.10$ \\
a Quaternion & double & $2.54 \pm 0.12$ & $2.60 \pm 0.03$ & $2.80 \pm 0.14$
\end{tabular}

\section{Appendix A. Experiments}

The experimental results are summarized in tables A.3 and A.4, and after these tables we explain how the experiments were performed. Table A.3 reports the ratio of the time taken by quotient algorithm and the time taken by scaling algorithm to execute the task mentioned in the first column. Each entry in Table A.3 is the average of a fairly large number of experiments, and the number after the \pm sign is the standard deviation.

\begin{tabular}{|c|c|c|c|c|}
\hline \multirow[b]{2}{*}{ Task } & \multirow[b]{2}{*}{ Precision } & \multicolumn{3}{|c|}{ Compiler/Operating system } \\
\hline & & $\begin{array}{l}\text { GCC } \\
\text { Linux }\end{array}$ & $\begin{array}{l}\text { Intel C++ } \\
\text { Linux }\end{array}$ & $\begin{array}{l}\text { Visual Studio } \\
\text { Windows }\end{array}$ \\
\hline Normalizing & single & $1.15 \pm 0.05$ & $1.04 \pm 0.02$ & $1.08 \pm 0.07$ \\
\hline a $2 \mathrm{D}$ vector & double & $1.17 \pm 0.02$ & $1.01 \pm 0.02$ & $1.00 \pm 0.05$ \\
\hline Normalizing & single & $1.38 \pm 0.05$ & $1.19 \pm 0.02$ & $1.22 \pm 0.09$ \\
\hline a $3 \mathrm{D}$ vector & double & $1.36 \pm 0.01$ & $1.08 \pm 0.53$ & $1.39 \pm 0.11$ \\
\hline Normalizing & single & $1.38 \pm 0.06$ & $1.89 \pm 0.13$ & $1.46 \pm 0.10$ \\
\hline a Quaternion & double & $1.42 \pm 0.08$ & $1.38 \pm 0.02$ & $1.66 \pm 0.09$ \\
\hline
\end{tabular}

The experiments used an Intel Core i7 2700K cpu with Ubuntu 14.04 LTS and an Intel Core i7 $950 \mathrm{cpu}$ with Windows 10 Pro, version 1511. The code for the experiments was written in $\mathrm{C}++11$ and was compiled with GCC 4.9.3 and Intel C++ 16.0.1 in Linux, with the -O3 optimizing flag (but not the flag -ffast-math.) In windows we used Visual Studio Enterprise 2015, version 14.0.25123.00, update 2, with the standard optimization flags for release builds. We tried to write simple code, with no effort to tune any algorithm to any machine. This code is available upon request to the author. Each entry in Tables A.3 and A.4 is the average over 500 experiments, and in each experiment we executed the algorithm $10^{6}$ times, in loops with a bit of extra code so that the optimizer would not remove the function calls that we wanted to time. We timed the loops without the function calls and subtracted the time for the empty loops from the ones we wanted to time. We measured the time with the functions clock_gettime in Linux and GetProcessTimesin Windows, and took into account only the time taken by the process. 


\section{Appendix B. Proofs}

In this section we prove Lemmas 1 and 2. Our proofs are based on [6], but one could obtain similar results using the theory in [3]. However, we note that [6] takes underflow into account whereas the standard model of floating point arithmetic in [3] does not, and dealing with underflow and overflow is the motivation for this article. As [3], we use $\mathrm{fl}(\operatorname{expr})$ to denote the computed value for the expression expr.

Our proofs use the following well known facts about IEEE floating point arithmetic, in which $x$ is a finite floating point number, $z \in \mathbb{R}$ and $\beta$ is a power of the base:

$$
\begin{aligned}
v \leq|\beta x| \leq \omega & \Rightarrow \mathrm{fl}(\beta x)=\beta x \\
|\beta x| \leq 2 v & \Rightarrow \mathrm{f}(\beta x)=\beta x+\delta \alpha \text { with }|\delta| \leq 1 / 2 \\
\mathrm{fl}(\beta x) & =\beta x+\delta \alpha \text { with }|\delta| \leq 1 / 2 \\
v \leq|z| \leq \omega & \Rightarrow|\mathrm{fl}(z)-z| \leq \frac{u}{1+u}|z| \\
|z| \leq 2 v & \Rightarrow \mathrm{fl}(z)=z+\delta \alpha \text { with }|\delta| \leq 1 / 2 \\
\mathrm{fl}(z) & =\gamma z+\delta \alpha \text { with }|\gamma-1| \leq \frac{u}{1+u} \text { and }|\delta| \leq 1 / 2 .
\end{aligned}
$$

We now state and proof some auxiliary results and after that we present the proofs of Lemmas 1 and 2.

Lemma 3. For $n=2,3,4$, let $\sigma$ and $\tilde{x}_{k}, k=1, \ldots, n$ be the numbers in Algorithms 5, 6 and 7 with input $x_{k}, k=1, \ldots, n$ and define $\check{x}_{k}:=\sigma^{-1} x_{k}$. If the $x_{k}$ are finite and $\sigma \neq 0$ then $\mathrm{fl}\left(\tilde{x}_{k}^{2}\right)=\mathrm{fl}\left(\check{x}_{k}^{2}\right)$.

Proof of Lemma 3 When $\sigma \neq 0$ we have that $\tilde{x}_{k}=\mathrm{fl}\left(\check{x}_{k}\right)$. Since $\sigma$ is a power of 2, if $\left|\check{x}_{k}\right| \geq v$ then $\tilde{x}_{k}=\mathrm{fl}(\check{x})=\check{x}_{k}$ because $\check{x}$ is a floating point number in this case. Therefore, $\mathrm{fl}\left(\tilde{x}_{k}^{2}\right)=\mathrm{fl}\left(\check{x}^{2}\right)$ when $|\breve{x}| \geq v$. If $|\check{x}|<v$ then Equation (6) and the monotonicity of rounding lead to $\left|\tilde{x}_{k}\right|=|\mathrm{fl}(\check{x})| \leq v$ and $0 \leq \mathrm{fl}\left(\tilde{x}_{k}^{2}\right), \mathrm{fl}\left(\check{x}^{2}\right) \leq \mathrm{fl}\left(v^{2}\right)=0$.

Lemma 4. For $n=2,3$ or 4 , assume that $\mathbf{x} \in \mathbb{R}^{n}$ and that Equations (5)-(10) hold. If we execute Algorithms 5, 6 or 7 with a finite input $\mathbf{x} \neq 0$ then the returned $\hat{r}$ and $\hat{\mathbf{x}}$ are such that $\hat{r} \neq 0$ and, for $r:=\|\mathbf{x}\|$ and $\overline{\mathbf{x}}:=\mathbf{x} / r$,

$$
\begin{aligned}
& \hat{\mathbf{x}}=\lambda(\overline{\mathbf{x}}+\mathbf{w}+\mathbf{z}) \quad \text { with } \quad|\lambda-1| \leq(2.001+n / 2) u, \\
& \left|w_{k}\right| \leq u\left|\bar{x}_{k}\right| /(1+u) \quad \text { and } \quad\|\mathbf{z}\| \leq 2 \sqrt{\alpha} u \text {. }
\end{aligned}
$$

If $(1+(1+n / 2) u) r \leq \omega$ then $\hat{r}$ is finite and

$$
2 r \geq 3 v \Rightarrow|\hat{r}-r| \leq(1+n / 2) r u
$$

and

$$
2 r \leq 3 v \Rightarrow|\hat{r}-r| \leq(1+n / 2) r u+\alpha / 2 .
$$

Finally,

$$
\|\hat{\mathbf{x}}-\overline{\mathbf{x}}\| \leq(3.001+n / 2) u
$$


Proof of Lemma 4 Let $\ell$ be an index such that $\left|x_{\ell}\right|=\max \left|x_{k}\right|$. In Algorithms 3, 4 and $7, \sigma \neq 0$ because $x_{\ell} \neq 0$. Let $\tilde{x}_{k}$ be the numbers in these algorithms and define

$$
s:=r^{2}, \quad \tilde{r}:=\|\tilde{x}\|, \quad \tilde{s}:=\tilde{r}^{2}, \quad \text { and } \quad \check{\mathbf{x}}:=\sigma^{-1} \mathbf{x} .
$$

Equations (5)-(10) imply that $\check{x}_{\ell} \in\left[\tau_{\min }, \tau_{\max }\right],\left|\check{x}_{\ell}\right| \in[v, \omega], \tilde{x}_{\ell}=\check{x}_{\ell}$ and $\tilde{x}_{k}^{2} \leq \tilde{x}_{\ell}^{2} \leq \omega / 8$. It follows that $v \leq \mathrm{fl}(\tilde{r})<\omega$ and Corollary 2 in [6] implies that

$$
\mathrm{fl}(\tilde{r}) \geq \mathrm{fl}\left(\sqrt{\tilde{x}_{\ell}^{2}}\right)=\left|\tilde{x}_{\ell}\right|=\sigma^{-1}\left|x_{\ell}\right| \geq \tau_{\min }
$$

Lemma 3 shows that

$$
\mathrm{fl}(\tilde{s})=\mathrm{fl}\left(\sigma^{-2} s\right) \geq \tau_{\min }^{2} \geq \alpha / u^{2},
$$

and the argument used in the proof of Corollary 11 in [6] and a bit of Algebra lead to

$$
\mathrm{fl}(\tilde{s})=\gamma_{s} \sigma^{-2} \quad \text { with } \quad\left|\gamma_{s}-1\right| \leq n u
$$

(Note that $n+1$ in [6] correspond to $n$ here.) As a result,

$$
\mathrm{fl}(\tilde{r})=\mathrm{fl}(\sqrt{\mathrm{fl}(\tilde{s})})=\gamma_{r} \sqrt{\gamma_{s}} \sigma^{-1} r \quad \text { with } \quad\left|\gamma_{r}-1\right| \leq \frac{u}{1+u} .
$$

The convexity/concavity arguments in [6] lead to

$$
\gamma:=\gamma_{r} \sqrt{\gamma_{s}} \quad \text { satisfies } \quad|\gamma-1| \leq(1+n / 2) u,
$$

and Equation (8) leads to

$$
\mathrm{fl}(\tilde{r})=\mathrm{fl}(\sqrt{\mathrm{fl}(\tilde{s})})=\gamma \sigma^{-1} r \leq 2 \gamma \sigma^{-1}\left|x_{\ell}\right| \leq 3 \tau_{\max } \leq 1 / \nu .
$$

If $3 v / 2 \leq r \leq \omega / \gamma$ then Equation (B.10) leads to

$$
\omega \geq \sigma|\mathrm{fl}(\tilde{r})| \geq 3 \gamma \nu / 2>v
$$

Equations (B.1) and (B.9) lead to

$$
0<\left|x_{\ell}\right| \leq \hat{r}=\mathrm{fl}(\sigma \mathrm{fl}(\tilde{r}))=\sigma \mathrm{fl}(\tilde{r}) \leq \omega,
$$

and Equation (B.10) shows that $|\hat{r}-r| \leq|\gamma-1| r$, and Equation (B.7) holds. The bound (B.8) follows from the argument above and Equation (B.5).

In algorithms 5, 6 and 7, $\tilde{x}_{k}=\mathrm{fl}\left(\sigma^{-1} x_{k}\right)$ and Equation (B.3) implies that

$$
\tilde{x}_{k}=\sigma^{-1} x_{k}+\rho_{k} \alpha \text { with }\left|\rho_{k}\right| \leq 1 / 2 .
$$

We also have $h=\mathrm{fl}(1 / \mathrm{fl}(\tilde{r}))$ and Equations (B.4) and (B.10) lead to

$$
h=\frac{\sigma \lambda}{r} \quad \text { with } \quad \lambda:=\gamma_{h} / \gamma=\frac{\gamma_{h}}{\gamma_{r} \sqrt{\gamma_{s}}} \quad \text { and } \quad\left|\gamma_{h}-1\right| \leq \frac{u}{1+u} .
$$


The usual upper bound on $\lambda=\gamma_{h} / \gamma$ is not a concave function of $u$, but since $u<10^{-6}$ we can prove that

$$
|\lambda-1| \leq(2.0001+n / 2) u,
$$

and this bound is a bit better than what is claimed by Lemma 4. Since $\hat{x}_{k}=\mathrm{fl}\left(h \tilde{x}_{k}\right)$ in all algorithms, Equations (B.6) and (B.11) yield

$$
\hat{x}_{k}=\lambda \gamma_{k}\left(\bar{x}_{k}+\rho_{k} \frac{\sigma \alpha}{r}\right)+\eta_{k} \alpha \text { with }\left|\gamma_{k}-1\right| \leq \frac{u}{1+u} \quad \text { and } \quad\left|\eta_{k}\right| \leq 1 / 2
$$

and $\hat{x}_{k}=\lambda\left(\bar{x}_{k}+w_{k}+z_{k}\right)$ with

$$
w_{k}:=\left(\lambda_{k}-1\right) \bar{x}_{k} \quad \text { and } \quad z_{k}:=\gamma_{k} \rho_{k} \sigma \alpha / r+\eta_{k} \alpha / \lambda .
$$

Since $r \geq\left|x_{\ell}\right|$ and $\tau_{\min } \geq \sqrt{\alpha} / u$ we have that

$$
\alpha \sigma / r \leq \alpha /\left(\sigma^{-1}\left|x_{\ell}\right|\right) \leq \alpha / \tau_{\min } \leq \sqrt{\alpha} u,
$$

and the bounds on $\gamma_{k}, \rho_{k}, \eta_{k}$ and $\lambda$ above and Equation (5) imply that $\|\mathbf{z}\| \leq 2 \sqrt{\alpha} u$. Finally, since $\|\overline{\mathbf{x}}\|=1$, the bounds above lead to

$$
\begin{gathered}
\|\hat{\mathbf{x}}-\overline{\mathbf{x}}\| \leq|\lambda-1|\|\overline{\mathbf{x}}\|+\lambda\|\mathbf{w}\|+\lambda\|\mathbf{z}\| \\
\leq(2.0001+n / 2) u+(1+(2.0001+n / 2) u) \frac{u}{1+u}+2 \lambda \sqrt{\alpha} u \leq(3.001+n / 2) u,
\end{gathered}
$$

and this completes the proof of Lemma 4.

Proof of Lemma 1 The only part of Lemma 1 which does not follow directly from Lemma 4 is the bound on $\phi$, which we now verify. Equation (B.13) and the bounds on $\lambda_{k}$ and $z_{k}$ in the proof of Lemma 4 lead to

$$
\|\hat{\mathbf{x}}\| \geq \lambda\left(1-\frac{u}{1+u}-2 \sqrt{\alpha} u\right)=\frac{\lambda}{1+u}(1-2(1+u) \sqrt{\alpha} u)
$$

and the usual formula for the cross product leads to

$$
\begin{gathered}
|\sin \phi|=\frac{\|\overline{\mathbf{x}} \times \hat{\mathbf{x}}\|}{\|\overline{\mathbf{x}}\|\|\hat{\mathbf{x}}\|} \leq(1+u) \frac{\|\overline{\mathbf{x}} \times(\mathbf{w}+\mathbf{z})\|}{1-2(1+u) \sqrt{\alpha} u} \leq(1+u) \frac{\|\mathbf{w}\|+\|\mathbf{z}\|}{1-2(1+u) \sqrt{\alpha} u} \\
\leq \frac{u+2(1+u) \sqrt{\alpha} u}{1-2(1+u) \sqrt{\alpha} u} \leq 1.001 u,
\end{gathered}
$$

and we are done.

Proof of Lemma 2 The only part of Lemma 2 which does not follow directly from Lemma 4 with $n=4$ is the bound on $q_{i} q_{j}$, which we now verify. Equation (B.13) and the bounds on $\lambda_{k}$ and $z_{k}$ in the proof of Lemma 4 lead to

$$
\hat{q}_{i} \hat{q}_{j}-\bar{q}_{i} \bar{q}_{j}=\left(\lambda^{2}-1\right) \bar{q}_{i} \bar{q}_{j}+\lambda^{2} \delta
$$


for

$$
\delta:=\bar{q}_{i}\left(w_{j}+z_{j}\right)+\bar{q}_{j}\left(w_{i}+z_{i}\right)+\left(w_{i}+z_{i}\right)\left(w_{j}+z_{j}\right) .
$$

Equation (B.12) with $n=4$ and the fact that $\|\mathbf{q}\|=1$ lead to

$$
\left|\left(\lambda^{2}-1\right) \bar{q}_{i} \bar{q}_{j}\right| \leq|\lambda-1||\lambda+1| \leq 4.0001 u(2+4.0001 u) \leq 8.001 u
$$

and

$$
\begin{gathered}
|\delta| \leq\|\overline{\mathbf{q}}\|\|\mathbf{w}+\mathbf{z}\|+(\|\mathbf{w}\|+\|\mathbf{z}\|)^{2} \leq\|\mathbf{w}\|+\|\mathbf{z}\|+(\|\mathbf{w}\|+\|\mathbf{z}\|)^{2} \\
\leq \frac{u}{1+u}+2 \sqrt{\alpha} u+\left(\frac{1}{1+u}+2 \sqrt{\alpha}\right)^{2} u^{2} \leq u .
\end{gathered}
$$

Therefore

$$
\lambda^{2}|\delta| \leq 1.001 u
$$

and the bound on $\left|\hat{q}_{i} \hat{q}_{j}-\bar{q}_{i} \bar{q}_{j}\right|$ follows from Equations (B.14) and (B.15).

\section{References}

[1] Altmann, S.L., Rotations, quaternions and double groups, Oxford University Press, 1986.

[2] Bindel, D.; Demmel, J.; Kahan, W.; Marques, O., On Computing Givens rotations reliably and efficiently. ACM Toms, 28 (2) 206-2008, 2002.

[3] Higham, N. J., Accuracy and stability of numerical algorithms, second edition, SIAM, 2002.

[4] IEEE Computer Society, IEEE Standard for Floating-Point Arithmetic, doi:10.1109/IEEESTD.2008.4610935. ISBN 978-0-7381-5753-5. 2008.

[5] Anderson, E.; Bai, Z.; Bischof, C.; Blackford, S.; Demmel, J.; Dongarra, J.; Du Croz, J.; Greenbaum, A.; Hammarling, S.; McKenney, A.; Sorensen, D., LAPACK Users' Guide (Third ed.). SIAM, Philadelphia, PA, 1999.

[6] Mascarenhas, W. F., Floating point numbers are real numbers, arXiv:1605.09202v1 [math.NA] 30 May 2016.

[7] AutoDesk inc., ObjectARX Developer's Guide, available at the Autodesk Developer Network, www.autodesk.com/developautocad. 